\title{
Quantum Perspectives of Consciousness, Cognition and Creativity: The Dirac Equation in a New Contour Integral Model of Brain Plasticity
}

Rossi EL* and Rossi KL

Director of the Psychosocial Genomics Institute, 125 Howard Avenue, Los Osos, CA. 93402, USA

\begin{abstract}
The quantum dynamics of consciousness, cognition and creativity are entangled in seemingly mysterious relationships with the classical Newtonian world view. Penrose, for example, believes that a completely new theory is required to integrate the classical and quantum perspectives on the royal road to reality. Mathematicians and physicists typically address such issues with questions about how cosmos and consciousness are related via the "Objective Reduction of the Quantum Wave Function" to Classical Newtonian Reality as people typically experience it. Aspects of the Penrose and Dirac quantum oriented perspectives of consciousness and cognition are integrated in this paper with recent RNA/DNA psychosocial genomic research in the classical-to-quantum and quantum-toclassical loops of information transduction that are proposed to account for so-called quantum weirdness as well as creativity and psychological crises in problem solving. Quantum perspectives are integrated with applications of the Dirac equation in a new contour integral model of brain plasticity, consciousness, cognition and creativity that could be utilized in translational medicine, psychotherapy and counseling. The development of new psychosocial genomic chips for documenting the loops of information transduction between consciousness and cognition to verbalization, RNA/DNA transcription, brain plasticity, new neural networks and the 4-stage creative cycle is recommended for facilitating further research on a quantum field theory of the human condition.
\end{abstract}

Keywords: Consciousness; Dirac equation; Evolution; Quantum; RNA/DNA; 4-Stage creative cycle; Novelty-numinosum-neurogenesis effect

\section{Introduction}

In his popular volume, "The Road to Reality: A Complete Guide to the Laws of the Universe," Penrose et al. [1] outlines a mathematical and physically oriented search for a new quantum perspective of cosmos, consciousness and cognition with these words. "Whereas it is commonly accepted that the appropriate quantum-gravity union must have been a major goal in the search for a fundamentally new perspective on physics, the message of these chapters is that we should seek a development in which the very rules of quantum (field) theory are not held sacrosanct but should be bent, just as should the geometry of our conventional space-time pictures. Nevertheless, there is clearly much math truth as well as beauty in quantum-mechanical principles, and these should not simply be abandoned. In twistor theory, instead of imposing QFT [Quantum Field Theory] rules, one looks into the rules and tries to extract features that mesh with those of Einstein's conceptions, seeking hidden harmonies between relativity and quantum mechanics. As has been stated earlier, one key element of guidance is the complex-number magic that has featured in so many places in this book. Another is an especial harmony with Einstein's theory of Lorentzian 4-space rather than with its generalizations to higher dimensions or to other signatures."

In two previous books, Penrose et al. [2,3] reviewed in great detail a "Search for the Missing Science of Consciousness" that dealt with many profound quandaries that are not resolved even today [4-7]. These issues are well expressed by Penrose, for example, in his questions about the "Natural Selection of Algorithms." "If we suppose that the action of the human brain, conscious or otherwise, is merely acting out some very complicated algorithm, then we must ask how such an extraordinarily effective algorithm actually came about. The standard answer, of course, would be natural selection. As creatures with brains evolved, those with more effective algorithms would have a better tendency to survive and therefore, on the whole, had more effective progeny. These progeny also tended to carry more effective algorithms than their cousins, since they inherited the ingredients of these better algorithms from their parents; so gradually the algorithms improvednot necessarily steadily, since there could have been considerable fits and starts in their evolution until they reached the remarkable status that we (would apparently) find in the human brain. "Even according to my own viewpoint, there would have to be some truth in this picture; since I envisioned that much of the brains action is indeed algorithmic ... I am a strong believer in the power of natural selection. But I do not see how natural selection, in itself, can evolve algorithms which could have the kind of conscious judgments of the validity of other algorithms that we seem to have." [2]. To deal some of these questions Penrose's math-oriented canon of cosmos and cognition [1-3] is integrated with the recent neuroscience-oriented epigenomic canon of consciousness and cognition, which has been generated over the past few decades $[8,9]$. Psychosocial genomics, a branch epigenomics, explains how nurture (memory and learning) modulates the expression of biological heredity in our DNA [10-13]. Epigenomics is the natural life process that supplements the limitations of early "natural selection theory" (Hughes, 2014). Psychosocial genomics, in particular, is an approach to answering the Penrose quandary about natural selection and the algorithmic nature of mind [14-16]. We begin with a review of RNA/ DNA psychosocial epigenomic research that underpins the molecular loop of information transduction between mind, neurons, genomics,

*Corresponding author: Rossi EL, Director of the Psychosocial Genomics Institute, 125 Howard Avenue, Los Osos, CA. 93402, USA, Tel:602-956-6196; E-mail: Ernest@ErnestRossi.com

Received July 21, 2014; Accepted August 12, 2014; Published August 18, 2014

Citation: Rossi EL, Rossi KL (2014) Quantum Perspectives of Consciousness, Cognition and Creativity:The Dirac Equation in a New Contour Integral Model of Brain Plasticity. J Appl Computat Math 3: 183. doi:10.4172/2168-9679.1000183

Copyright: @ 2014 Rossi EL, et al. This is an open-access article distributed under the terms of the Creative Commons Attribution License, which permits unrestricted use, distribution, and reproduction in any medium, provided the original author and source are credited. 
brain plasticity and experiences of new consciousness and cognition.

\section{Dirac's Equation and a Contour Integral Model of Brain Plasticity and Consciousness}

We carefully follow Dirac's own concise mathematical reasoning [17] in this presentation. Only our psychological application of Dirac's et al. [18] quantum field theory for creating our new contour integral model of brain plasticity, cognition and consciousness is original here. We are certainly aware that this application of the quantum microworld of Planck's constant to the macroscopic level of human cognition and consciousness may violate fundamental assumptions of quantum field theory. We therefore present the following as a Penrose thought experiment that will require considerably more empirical research as outlined in the next section before it could be taken seriously.

Dirac begins with the idea of Schrödinger's wave function in 3-dimensional space. The wave function for some particle, here designated as $\psi$, is a function of the three coordinates $\mathrm{x}_{1}, \mathrm{x}_{2}, \mathrm{x}_{3}$ that can vary with time:

$$
\psi\left(\mathrm{x}_{1}, \mathrm{x}_{2}, \mathrm{x}_{3} ; t\right) .
$$

Dirac notes that the usual interpretation of this wave function when normalized is that the square of its modulus $|\psi|^{2}$ providing the probability of the particle being a particular place. This wave function $\psi$ is a complex number so it can be multiplied it by its phase factor $\mathrm{e}^{\mathrm{i} \gamma}$, where $\gamma$ is a real number so that $\mathrm{e}^{\mathrm{i} \gamma}$ has a modulus of unity. Dirac then multiplies $\psi$ by $\mathrm{e}^{\mathrm{i} \gamma}$ to get another wave function, here designated as $\Psi$ :

$$
\mathrm{e}^{\mathrm{i} \gamma} \psi \equiv \boldsymbol{\Psi}
$$

Which now has its modulus squared just as $\psi$ :

$$
|\Psi|^{2}=|\psi|^{2} \text {. }
$$

This allows $\Psi$ and $\psi$ to have the same probability distribution.

Dirac then notes that $y$ in equation (2) could be a function of position as well as time so that the new $\Psi$ has the same probability distribution as $\psi$ in equation (4):

$$
\Psi\left(\mathrm{x}_{1}, \mathrm{x}_{2}, \mathrm{x}_{3} ; t\right)=\mathrm{e}^{\mathrm{i} \gamma(\mathrm{x}, \mathrm{x}, \mathrm{x} ; t)} \begin{gathered}
12 \\
2
\end{gathered}
$$

However, the new $\Psi$ and the original $\psi$ do not satisfy the same wave equation! This is becomes evident when Dirac forms $\partial \Psi / \partial \mathrm{x}_{\mathrm{r}}$ with $r$ taking on the values of 1,2 or 3 so that he obtains equation 5:

$\partial \Psi / \partial x_{\mathrm{r}}=\mathrm{e}^{\mathrm{i} \gamma}\left(\partial / \partial x_{\mathrm{r}}+i K_{r}\right) \psi$

Where $\mathbf{K}_{\mathbf{r} \text { is }}$ a function of position in equation 6 :

$\mathbf{K}_{\mathbf{r}} \equiv \partial \gamma / \partial \mathbf{x}_{r^{*}}$

Dirac then states "We would have to consider $\mathrm{K}_{\mathrm{r}}$ as something more general, something such that when we take $\mathbf{K}_{\mathrm{r}} \mathrm{dx}_{\mathrm{r}}$ and integrate around a closed loop, the result need not be zero:

$$
\oint \mathrm{K}_{\mathrm{r}} \mathrm{dx}_{\mathrm{r}} \text { need not be equal to } 0 \text {. }
$$

Dirac then concludes "If we do that, we get a physical theory which is definitely more general than what we had before". Dirac's comment that the contour integration of equation 7 "need not be equal to 0" implies how new awareness could arise from a cycle of brain plasticity and new cognition as outlined in the next section. This suggests how our new contour integral model of brain plasticity and consciousness together with Dirac's quantum field theory could provide a satisfactory answer to the Penrose quandary about natural selection and the algorithmic nature of mind outlined above. In this new application of $[19,20]$ original description of the electron to our RNA/DNA psychosocial genomic theory of cognition, consciousness and creativity we are following [21] deep insight into the nature of mathematical equations as articulated by Hertz.

"One cannot escape the feeling that these mathematical formulae have an independent existence and an intelligence of their own, that they are wiser than we are, wiser even than their discoverers that we get more out of them than was originally put into them.

\section{Heinrich Hertz, on Maxwell's Equations of Electromagnetism}

It is precisely the "feeling that these mathematical formulae have an independent existence and an intelligence of their own" that motivates our thought experiment of using Dirac's equation originally developed on the quantum level to the macro level of RNA/DNA transcription and translation in our Psychosocial Genomic Theory of Cognition, Consciousness and Creativity.

\section{The RNA/DNA Psychosocial Genomic Theory of Cognition, Consciousness and Creativity}

We now propose how Dirac's approach to quantum field theory could be applied to the RNA/DNA psychosocial genomic theory of consciousness and cognition illustrated in Figures 1, 2 and 3. We previously documented how Hadamard (1945) summarized Poincaré's 4-stage creative cycle (data collection, incubation, insight, verification) corresponds to the 4-stages of the psychobiological cycles of Figures 1 ,

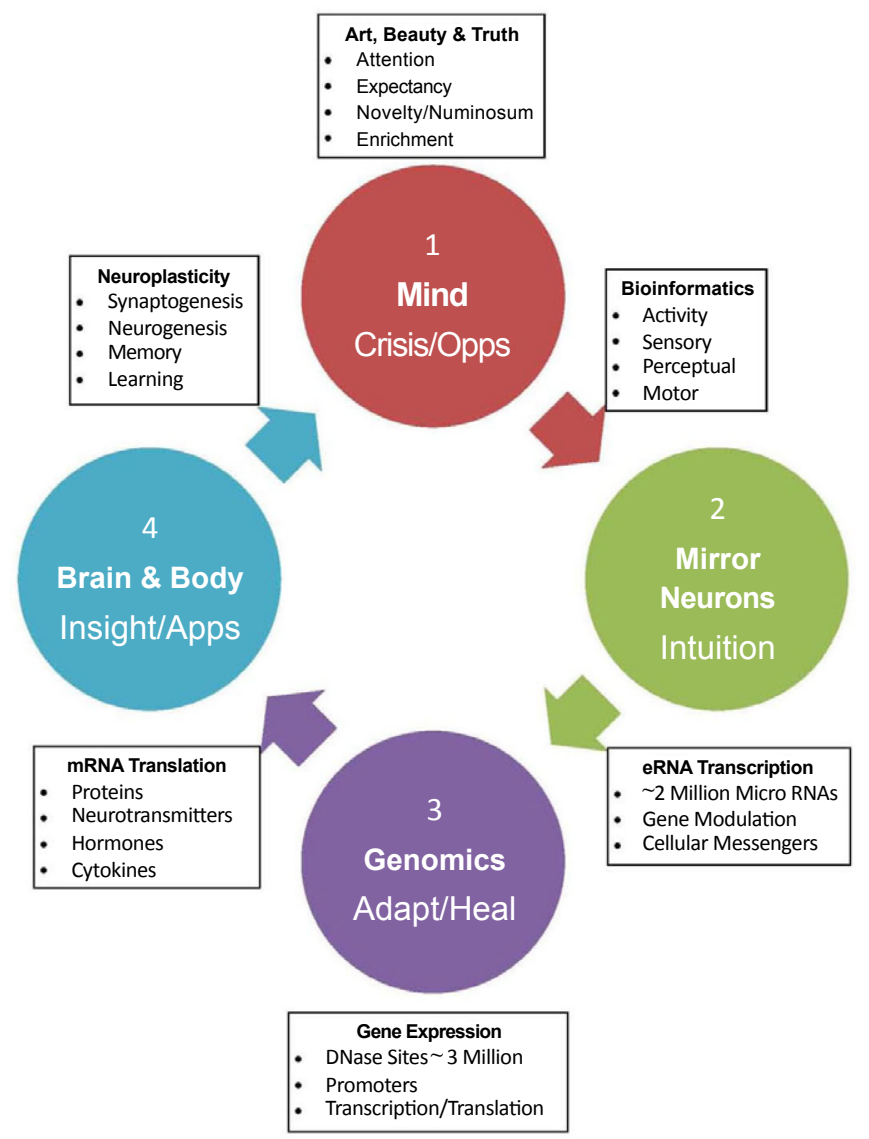

Figure 1: The psychosocial genomic cycle of consciousness, cognition and brain plasticity continually updated by the daily and hourly 4 -stage creative cycle $[15,16,22]$ 


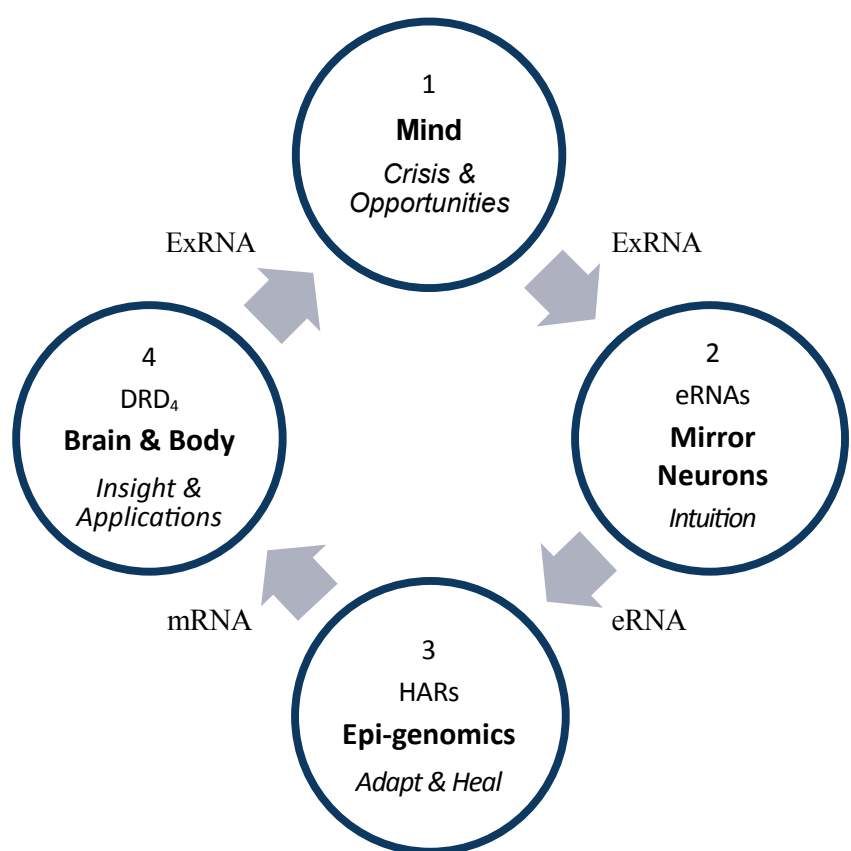

Figure 2: An update of the Watson/Crick central dogma of molecular biology. Cognitions modulate gene expression via the bioinformatics epi-genomic loop mediating between nature and nurture. Cognitions are converted into eRNAs (enhancer RNAs) to enhance DNA (gene expression), which codes for mRNAs (messenger RNAs) that generate the proteins (hormones, neurotransmitters, cytokines, etc.) throughout the expanded complex adaptive mirror neuron system that underpins mind/body communication $[9,10,12,79]$

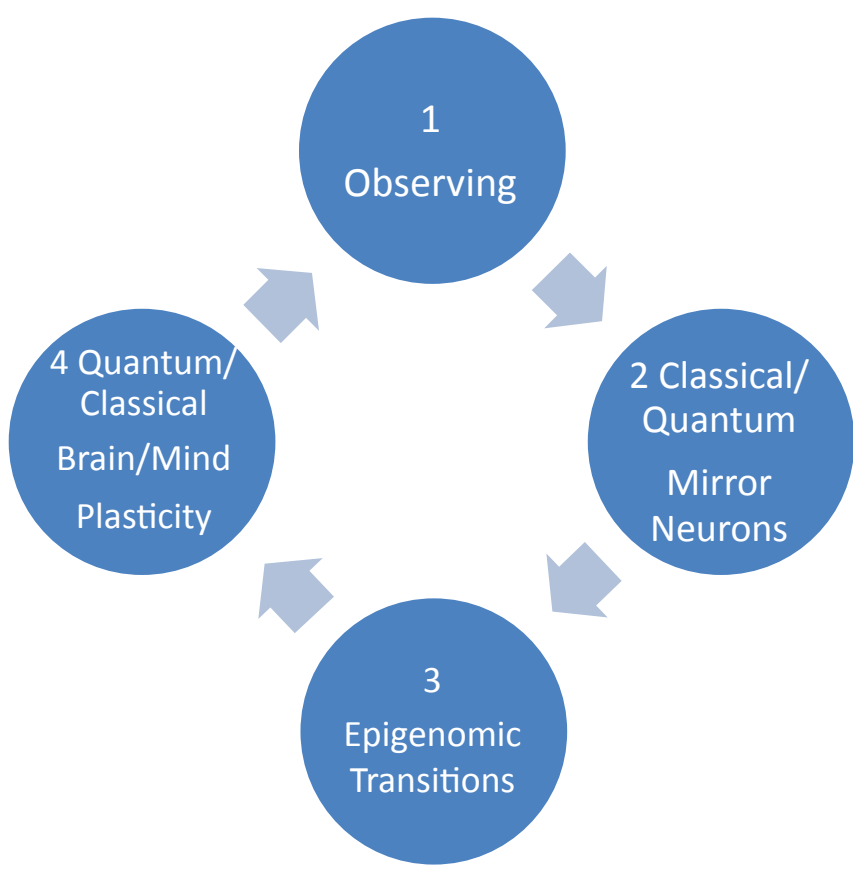

Figure 3: The Communication Cycle between 1. Observing Consciousness, 2. The Classical-to-Quantum Dynamics of Mirror Neurons, 3. Epigenomic Transitions and 4.The Quantum-to-Classical Dynamics on the Road to Constructing New Cognition, Consciousness and Reality.

2 and $3[9,12,13,15]$. Figure 1 , for example, is a very broad functional definition of the gene as a complex adaptive system that embraces the daily and hourly cycles of life on all levels from mind to DNA [22].

\section{The Mind/Gene Communication Cycle of Molecular Biology}

The top circle of Figure 1 embraces the classical experimental research of historical psychology updated with the more recent bioinformatics and neuroscience research embracing art, beauty, creativity, music, truth, and dreams [5,23]. Research at this top level of consciousness, cognition, imagination and dreaming manifests the novelty-numinosum-neurogenesis-effect as an adaptive complex motivational system [24]. Focused consciousness, attention and novelty evokes highly motivating experiences of the numinosum (fascination, mysteriousness, tremendousness, $[25,26]$ that activate neurons to turn on gene expression via the Novelty-Numinosum-Neurogenesis-Effect (NNNE), which was originally described in this way [9]. "It is not so much what is expected and easily predictable in human affairs that is motivating but the exact reverse! That which is surprising, unknown and unexpected garners our attention and sets us forth on human quests for adventure in the novelty-numinosum-neurogenesis experiences of love and life as well as the arts and sciences... Novel interactions between the organism and the environment evoke [activity-dependent] gene expression, protein synthesis, neurotransmitters and neurogenesis operate via the Darwinian principles of natural variation and selection on all levels from the molecular genomic to the subjective states of consciousness. In essence we propose that the Novelty-NuminosumNeurogenesis-Effect is a top-down process of generating adaptive states of consciousness and cognition $[9,12,13,27]$, which are integrated with the typical bottoms-up dynamics of molecular biology characteristic of most life cycles [22]. Key research is now exploring how these cyclic complex adaptive life systems of the transcription/translation dynamics arising from $\sim 2$ million eRNAs transducing information from the physical environment and psychosocial milieu to genes bearing $\sim 3$ million docking sites recently summarized by the ENCODE Consortium [28]. In the past decade DNA microarray technology has made it possible to measure the expression levels of many thousands of genes simultaneously [29]. This evidence-based research in molecular biology has become a new standard in personalized medicine. Current research documents the use of DNA microarrays for assessing therapeutic responses via a variety of top-down psychogenomic processes that were originally developed by many diverse cultural, historical and spiritual traditions of mind-body healing. These include the relaxation response [30], therapeutic hypnosis [13,15,31-33], meditation [34], the therapeutic placebo [35], social psychology [36-40] and yoga [41] to facilitate optimal states of human consciousness and cognition as well as the healing of stress related dysfunctions [42]. We extended this use of DNA microarrays to explore the hypothesis that such topdown therapeutic protocols epitomized by The Psychosocial Genomic Healing Experience (CPGHE) can be the foundation of a more general theory of mind-body communication and healing. A full description of the administration, scoring and successful clinical application of the top-down CPGHE protocol is freely available [13]. We propose that this CPGHE protocol could update the cognitive-behavioral efficacy of evidence-based translational medicine recommended as a standard of clinical excellence by Insel et al. [43-45], Director of National Institute of Mental Health.

\section{The Classical-to-Quantum Transition via Mirror Neurons}

The original research on mirror neurons initiated by Rizzolatti et al. [46] and others [47-50] Regev et al. has been greatly expanded in current neuroscience to include epigenomic processes that modulate mind/gene communication. Research on bird song courtship dynamics, for example, documented how eRNAs ("enhancer RNAs" enhance gene expression) respond to thought by modulating the 
transcription/translation cycle via activity-dependent epigenomic expression. Clayton, a specialist in songbird neurogenomics made the salient comment, "this is the first time a microRNA has been shown to respond to a particular thought process" [51-55].

Epigenomic information transduction between the sound spectra of the bird song and molecular eRNAs is encoded via our expanded definition mirror neuron activity. It is fascinating to learn, for example, that a young male finch learns his song by trying to imitate his father. Alas, however, the young fellow invariably makes "mistakes." Yet these so-called "mistakes" become an aspect of his individual identity which apparently pleases a young female who then chooses to mate with him. The male's song and the female's choice behavior is associated with turning on the molecular-genomic RNA/DNA transcription/ translation cycle leading to the appropriate production of hormones, ovulation and sexual behavior in the lady. More recent research on the social communication of bats confirms and extends this earlier research on bird songs. Since bats are mammals their songs provide greater detail about their appropriateness as a model of human cognition. A recent issue of Science [56] details how the FOXP2 gene, which is associated cognition and vocal learning in humans, birds and bats, may be a closer model for human speech. The trills, chirps and buzzes of bats, for example, can communicate a series of cognitions such as announcing (1) I am species P. Nathusii, (2) a male, (3) specifically I am male $17 \mathrm{~b}$ (females learn to recognize individual song of males with whom they choose to mate, (4) land here next to me, (5) we share a common social identity and communication pool, (6) soft tones lure ladies while (7) harsh tone compete with other males and warn them away. Although these songs typically last only $\sim 1.6$ seconds they may contain $\sim 20$ syllables combined in specific ways with individual rhythmic patterns of communication that are appropriate for current life conditions $[57,58]$.

Such research in bird and bat song syntax and semantics mediates the vastly more complex analogous cycle of information transduction that occurs in human consciousness and cognition as illustrated in Figures 1, 2 and 3 . This is the fundamental insight that integrates the top-down path of mind, consciousness, and the behavioral intentions of so-called "free will" with the bottoms-up molecular-genomic paths of communication within and between individuals. We now speculate that this is a manifestation of the quantum operators engaging entanglement to bridge the so-called "Cartesian gap" between mind and body-between the physical mass of cosmos and the apparently massless light of human consciousness [59] Illustrated in Box 1.

Free public data bases are being updated daily by the National Institute of General Medical Sciences, which offers information on these advances of The New Genetics. Francis Collins, Director of the National Institutes of Health (NIH) is now funding research on their new Extracellular RNA (ExRNA) Communications Program. The NIH Common Fund provides research grants to catalogue all types of ExRNA that flow between mind and body "in blood, tears, saliva and every other body fluid" to provide a baseline that can be compared with ExRNA profiles associated with Alzheimer's, ageing, autism, diabetes, obesity, psychiatry, Parkinson's, stress, trauma, etc. [60]. These research developments illustrated in Figure 2 lead us to propose how the psychosocial epigenomics of ExRNAs signaling between nature and nurture are one of the many classical molecular/genomic systems underpinning of the complex adaptive dynamics of the cognition, consciousness and creativity.

\section{Experience-dependent gene expression illustrates theepigenomic level in Figure 2}

Pollard et al. [61] has pioneered research into the Human Accelerator Regions (HARs) that are now recognized as being groups of genes that are undergoing very rapid adaptation distinctively different from our nearest primate relatives. Notice how we give precise bioinformatics meaning to the commonly used but vague psychological terms of the 4-stage creative process such as "Crisis/ Opportunity" (stage 1), "Intuition" (stage 2), "Adaption/Healing" (stage 3) and "Insight/Applications" (stage 4) [13,15,16,62]. The implications of this epigenomic general theory of mind-body communication range from the practical, such as the development of more effective psychiatric drugs and psychotherapy, to the profoundly philosophical issues of integrating mind and matter in our conceptions of the cosmos, cognition and consciousness. Brain/body research has a new psychogenomic foundation in the translational process of coding for mRNAs, proteins at the molecular-genomic level. Key research explores how these proteins, often called "mother molecules," are cleaved into the neurotransmitters, hormones, and cytokines of the complex adaptive system of psychoneuroimmunology which integrate cells of the mind, brain and body that ultimately facilitate the dynamics of memory, learning, behavior, cognition and consciousness itself via synaptogenesis and neuroplasticity, etc. [15].

\section{Brain/Mind Plasticity and the Quantum to Classical Transition}

Figure 3 illustrates the epigenomic transitions between classicalto-quantum dynamics in stage 2 and quantum-to-classical dynamics in stage 4 of the creative cycle of the RNA/DNA psychosocial genomic theory of cognition and consciousness. It is interesting to ask, for example, whether the intuitions of stage 2 are psychologically sensed or felt to be the same or different in comparison with the experience of insights during stage 4 . This is a particularly important issue because we presume that Stage 4 of the creative cycle is the quantum-to-classical transition that purportedly takes place in the Penrose/Hameroff"Orch OR" model of consciousness entangled with microtubule structure and function within the cells of brain [63]. We now propose it is precisely during the classical-to-quantum (stage 2) and quantum-to-classical (stage 4) transitions that people typically experience psychological difficulties that sometimes become severe enough to be described as a "crisis as illustrated in Figures 4 and 5. Jeong, Lim and Kim et al. [64] recently investigated the dynamics classical-to-quantum and quantum-to-classical transitions in a manner that is consistent with the psychosocial genomic perspective of Figure 4 . Such implicit brain/mind plasticity is typical of the Stage 2 (classical-to-quantum) and stage- 4 (quantum-to-classical) phase transitions of creative consciousness and cognition illustrated in Figure 3. We speculate that such quantum/ classical transitions could function as natural inner resources for problem solving $[11,14,15,65,66]$. This implies how a broad application of the complex-valued four-component field of Dirac's Equation illustrated by Mutze et al. in Box 1 could become a new model of the 4-stage creative cycle as it is experienced in the arts and sciences as well as problems in everyday life [50,68-71]. Figures 4 and 5 also illustrate the emotional crises associated with difficult classical/quantum and quantum/classical phase transitions.Hot or intense phases of high performance activities during the 4-stage creative process are illustrated in red in the top part of Figure 6. These red high performance peaks alternate with low phases of healing and recovery shown in green during the 90-120 minute basic rest-activity cycle. The "Breaking Point" in the late afternoon is an important circadian period of transition wherein 
Citation: Rossi EL, Rossi KL (2014) Quantum Perspectives of Consciousness, Cognition and Creativity:The Dirac Equation in a New Contour Integral Model of Brain Plasticity. J Appl Computat Math 3: 183. doi:10.4172/2168-9679.1000183

Page 5 of 8

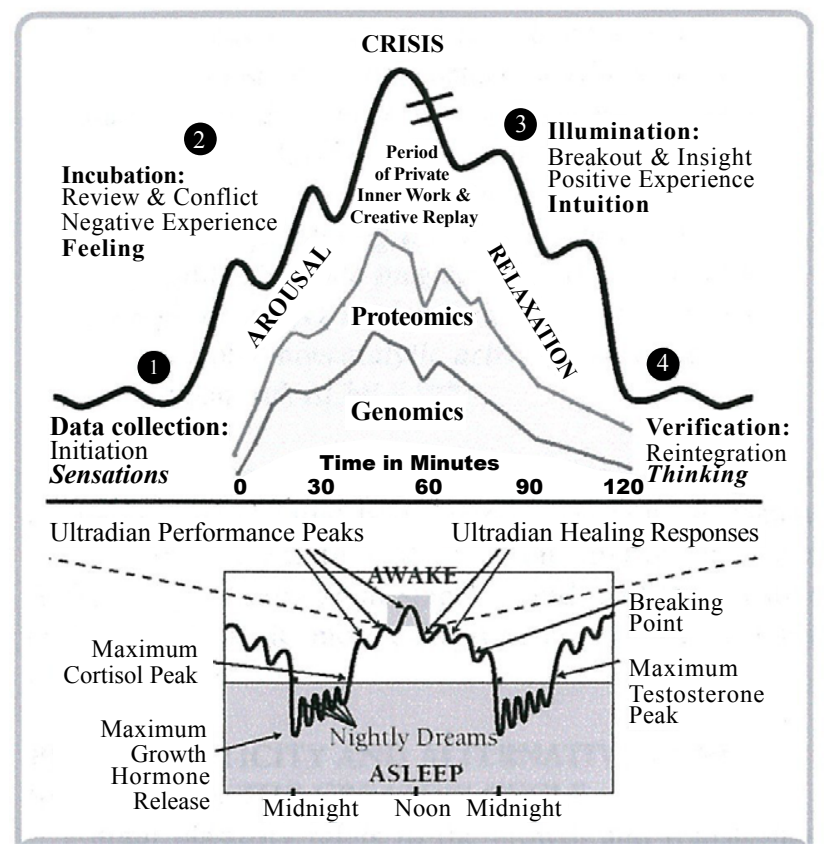

Figure 4: A profile of the 4-stage creative process attributed to Henri Poincaré [62] as a basic paradigm of our RNA/DNA psychosocial genomic theory of mind/ body stress experienced in the 90-120 minute basic rest-activity cycle during everyday life, sleep, dreams and psychotherapy [15,16,80-83].

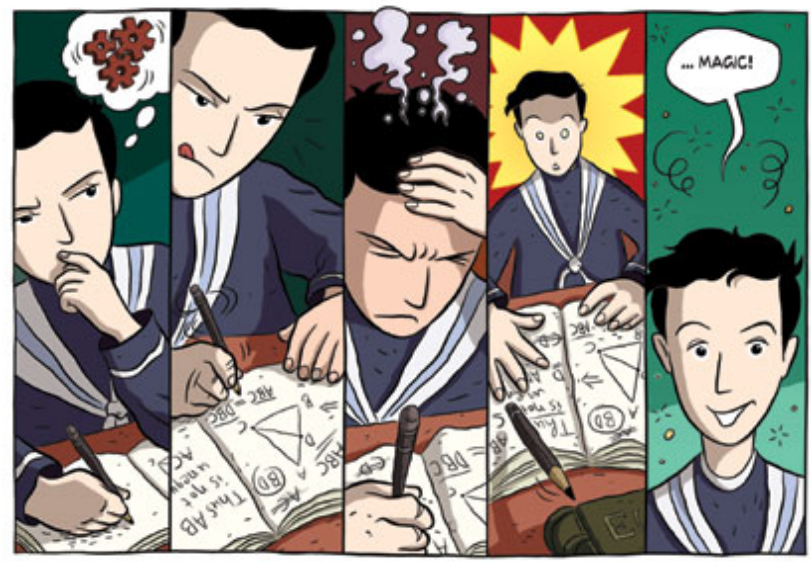

Figure 5: A cartoon of the 4-stage creative cycle illustrating the typical stress and crisis experienced as a psychological aspect of problem solving [84].

there is a normal shift in gene expression and behavior changing from an outer world performance orientation to an inner world of satisfying recovery in sleep and dreaming [22]. The bottom part of Figure 6 illustrates the recent research of Xie et al. [72] documenting the cleaning up of toxic metabolic waste products of daily work cycles during sleep (green) and dreaming (rainbow). The small rainbows support our hypothesis of how the presence of many such alternating phases of RNA/DNA activity during waking consciousness as well as REM dreaming also clean up toxic waste products of the performance peaks brain/mind metabolism. Current neuroscience research implies how this could be the molecular/genomics underpinning of many holistic psychotherapeutic practices that utilize rest and relaxation (therapeutic hypnosis, prayer, meditation, yoga, etc.) developed over the ages by many cultures $[15,16,73,74]$. The alternating phases and epigenomic entanglements of creativity, cognition and consciousness illustrated herein appear to be manifestations the basic quantum wave nature of many natural psychobiological processes. Considerable research is now required to assess the degree such wave patterns of nature in human behavior, cognition and dreaming are consistent with our RNA/DNA psychosocial genomic theory of the contour integral theory of the quantum nature of brain plasticity, cognition and consciousness that could be utilized in translational medicine, counseling, psychotherapy.

\section{Discussion: Current Controversies of Brain and Consciousness Research in Historical Perspective}

A modicum of data may throw some light on current controversies about the quantum nature of the brain and consciousness research priorities of this paper in historical context [75-77] as well as "A call

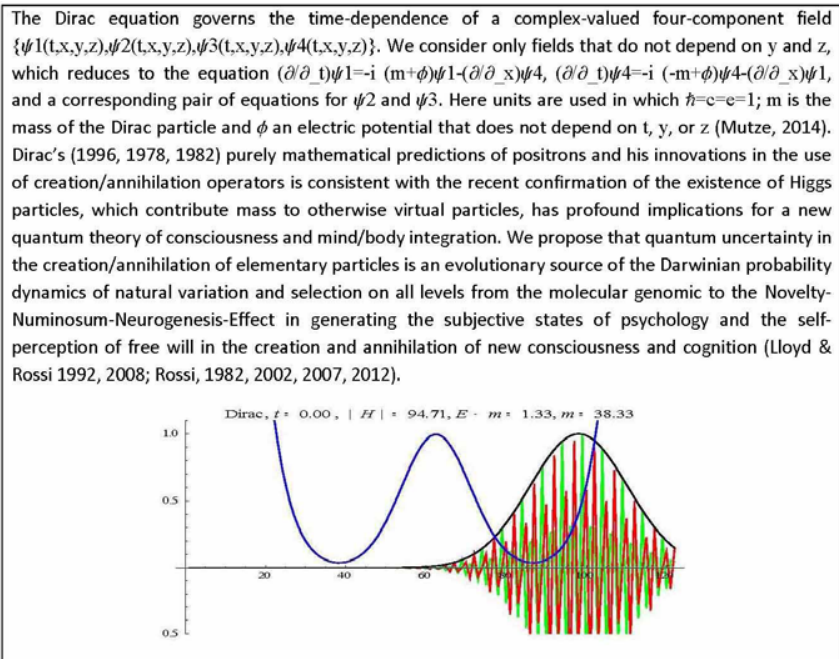

Box 1: The Dirac Equation: Quantum Dynamics as a Model of the 4-Stage Creative Cycle on all Levels from Mind to Gene.

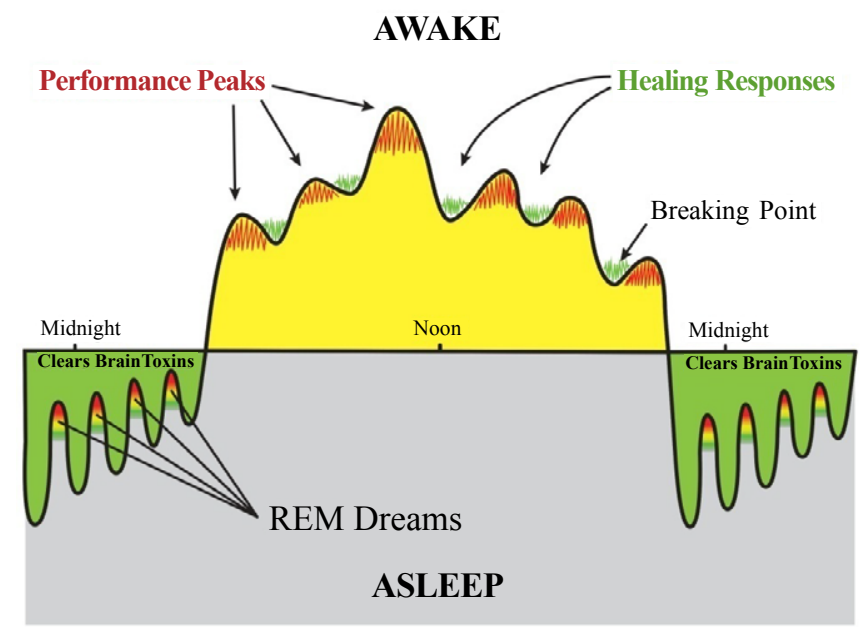

Figure 6: A profile of our RNA/DNA psychosocial genomic theory of Poincare's 4-stage creative process during the 90-120 minute basic rest-activity cycle during everyday life, sleep and dreams. 


\section{TRANSFORMATION Of CONSCIOUSNESS} A 200 YEAR PSYCHOLINGUISTIC STUDY

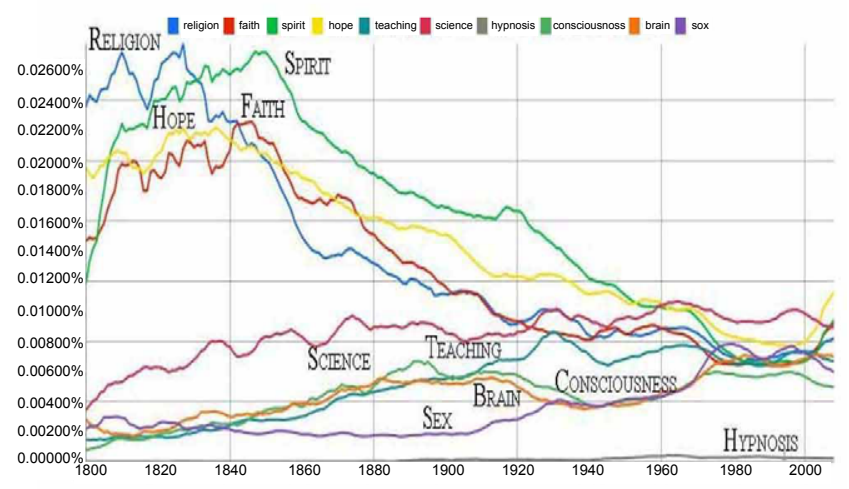

Figure 7: A Google n-gram comparing the frequencies of English words like 'religion, spirit, faith and hope,' which have been gradually losing their digita psycholinguistic frequency over the past 200 years while words like 'science, teaching, sex, brain and consciousness' have been gaining.

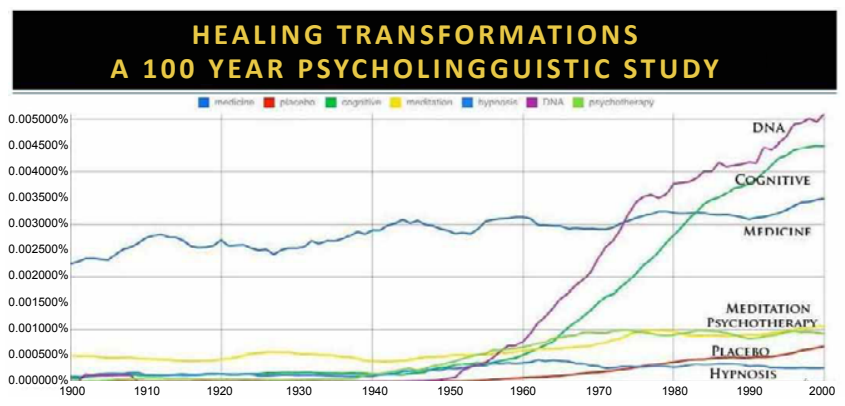

Figure 8: A Google n-gram of the psycholinguistic frequencies of a number of therapeutic approaches to healing that appear to have linear growth in comparison with the exponential growth of "DNA" and the "cognitive" since the 1960s

for mental health science" [77]. The two transformational graphs of cultural and scientific consciousness presented in Figures 7 and 8 are examples of the new discipline of the digital humanities - the use of computer algorithms to search for meaning in large databases of text and media. This new digital discipline was used to explore 200 years of the cultural history of the transformations of consciousness in over five million digitized books from more than 40 university libraries around the world. Figure 7 is a Google n-gram graphically comparing the frequencies of English words like 'religion, spirit, faith and hope,' which have been gradually losing their digital linguistic frequency over the past 200 years while words like 'science, teaching, sex, brain and consciousness have been gaining. What can this mean? The first n-gram documents our 200 year psycholinguistic history of the emergence of science out of traditional faith based ideologies. A careful examination of the lower right-hand side of this graph, however, suggests that an asymptote is being formed since the 1960's leading to a fairly even balance of the faith based mentality with the scientific at the present time. Of course, a continuing competition between these two perspectives could be expected to generate the current controversies about the Human Brain Project [75,76]. Current consciousness on the lower right hand side of this 200 year history appears to be an emerging integration of traditional spiritual values with modern education and science that could give both sides room for hope. Figure 8 is a more recent 100 year history of human quests for the meaning and an understanding of life on all levels that to justifies the current "A call for mental-health science" [77]. Once again something dramatic seems to breaking through since the 1960's: "DNA" and the "Cognitive" appear to be leading to the future with exponential growth curves that seem to be leaving behind the more leisurely linear paths of healing transformations here represented by the words "medicine, meditation, psychotherapy, hypnosis" and the ever present "placebo."

Rather than leaving medicine, meditation, psychotherapy, hypnosis and placebo behind, however, we prefer the more positive possibility that innovative Top-Down research combining DNA with the Cognitive as well as the Bottoms-Up of traditional molecular biology will underpin the efficacy of the healing transformations of all cultures. This is the motivation for many new open access scientific publications with bioinformatics themes on the internet such as the Journal of Psychosocial and Cultural Genomics that integrate the cyclic Top-Down/Bottoms-Up perspectives [31]. Current research by the Psychiatric Genomics Consortium [78], for example, recently has developed a Psycho Chip, which identifies parts of the human genome associated with a variety of mental illnesses such as Alzheimer's, Parkinson's, schizophrenia, autism, anorexia nervosa, bipolar and obsessive-compulsive disorders. A similar Psycho Chip is now needed to assess the normal psychosocial and cultural epigenomic RNA/DNA dynamics of the 4-stage creative cycle as they are taking place in real time in everyday life and creative states of optimal performance.

\section{Summary}

Quantum concepts of consciousness, cognition and creativity are integrated with the classical Newtonian world view as people ordinarily experience it. Current research in neuroscience is reviewed to underpin a new RNA/DNA psychosocial genomic theory of the classical/quantum transformations of human behavior, cognition and consciousness. Alternating classical-to-quantum and quantum-to-classical transitions on all levels from mind to gene are explored in practical application of Dirac's quantum field theory and mathematical notation for the Human Brain Project and mental health. Quantum perspectives are integrated with Dirac's equation in a new contour integral model of brain plasticity, consciousness and cognition as they are experienced during alternating periods of creativity, problem solving and stress in the arts, humanities and sciences as well as everyday life, counseling, psychotherapy and translational medicine. Quantum uncertainty in the creation/annihilation of elementary particles is proposed as an evolutionary source of the Darwinian probability dynamics of natural variation and selection on all levels from the molecular genomic to the Novelty-Numinosum-Neurogenesis-Effect in generating the subjective states of psychology and the self-perception of free will in the creation/annihilation of new states of consciousness and cognition. The development of new PsychoChips for documenting optimal loops of information transduction from consciousness and cognition to verbalization, RNA/DNA transcription/translation dynamics, brain plasticity, neural networks and the 4 -stage creative cycle is recommended for facilitating further research on a quantum field theory of the human condition.

\section{References}

1. Penrose R (2004) The Road to Reality: A Complete Guide to the Laws of the Universe. NY: Knopf.

2. Penrose R (2002) The Emperor's New Mind: Concerning Computers, Minds and the Laws of Physics. Oxford: Oxford University Press. 
Citation: Rossi EL, Rossi KL (2014) Quantum Perspectives of Consciousness, Cognition and Creativity:The Dirac Equation in a New Contour Integral Model of Brain Plasticity. J Appl Computat Math 3: 183. doi:10.4172/2168-9679.1000183

Page 7 of 8

3. Penrose R (1996) Shadows of the Mind: A Search for the Missing Science of Consciousness. NY: Oxford University Press.

4. Berry M (1998) Paul Dirac: The purest soul in physics. Physics World.

5. Dehaene S (2014) Consciousness and the Brain: How the Brain Codes Our Thoughts. NY: Viking

6. Dirac P (1982) Principles of Quantum Mechanics. (4th edn). Oxford, England Oxford University Press.

7. Dresden M (1990) Dirac A Scientific Biography. HelgeKragh. Cambridge University Press, New York, 1990. x, 389 pp., illus. \$44.50.Science 249: 937.

8. Rossi E (1993) The Psychobiology of Mind-Body Healing: New Concepts of Therapeutic Hypnosis. ( $\left.2^{\text {nd }} e d n\right)$ NY: WW Norton.

9. Rossi E (2002) The Psychobiology of Gene Expression: Neuroscience and Neurogenesis in Hypnosis and the Healing arts. NY: WW Norton.

10. Rossi E (2004) A Discourse with Our Genes: The psychosocial and cultural genomics of therapeutic hypnosis and psychotherapy. Zeig, Tucker and Theisen.

11. Rossi E (2005) Einstein's eternal mystery of epistemology explained: The four stage creative process in art, science, myth, and psychotherapy. Annals of the American Psychotherapy Association 8: 4-11.

12. Rossi E (2007) The Breakout Heuristic: The New Neuroscience of Mirro Neurons, Consciousness and Creativity in Human Relationships. The Selected Papers of Ernest Lawrence Rossi, Erickson Foundation Press.

13. Rossi E (2012) Creating Consciousness: How Therapists Can Facilitate Wonder, Wisdom, Truth and Beauty. The Selected Papers of Ernest Lawrence Rossi, Erickson Foundation Press.

14. Rossi E, Rossi K (1996) The Symptom Path to Enlightenment: The New Dynamics of Hypnotherapeutic Work: An Advanced Manuel for Beginners. NY: Zeig, Tucker, Theisen.

15. Rossi E, Rossi K (2013) Creating New Consciousness in Everyday Life: The Psychosocial Genomics of Self-Creation. Los Osos, CA: The Psychosocial Genomic Institute of the California Central Coast.

16. Rossi E, Rossi K (2014) An Evolutionary RNA/DNA Psychosocial Genomics Theory of the Transformations of Consciousness: The Quest for Therapeutic Mind/Gene Search Algorithms. The International Journal for Transformations of Consciousness 1: 1-20.

17. Dirac P (1978) Directions in Physics. NY: Wiley.

18. Dirac P (1966) Lectures on Quantum Field Theory. NY: Yeshiva University.

19. Dirac P (1928a) The Quantum Theory of the Electron. Proc Roy Soc London.

20. Dirac P (1928b) The Quantum Theory of the Electron, Part II. Proc Roy Soc London.

21. Wilczek F (2002) A Piece of Magic: The Dirac Equation. It Must be Beautiful: Great Equations of Modern Science. 102-130.

22. Lloyd D, Rossi E (1992) Ultradian Rhythms in Life Processes: An Inquiry into Fundamental Principles of Chronobiology and Psychobiology. NY: SpringerVerlag

23. Kandel E (2006) In search of memory: the emergence of a new science of mind. NY: WW Norton.

24. Holland J (2012) Signals and Boundaries: Building blocks for complex adaptive systems. Cambridge, Massachusetts: MIT Press.

25. Otto R (1923) The Idea of the Holy. London: Oxford University Press.

26. Jung C (1958) Psychology and Religion: West and East. NY: Pantheon Books.

27. Rossi EL (1982) Hypnosis and ultradian cycles: a new state(s) theory of hypnosis? Am J Clin Hypn 25: 21-32.

28. ENCODE Project Consortium (2012) An integrated encyclopedia of DNA elements in the human genome. Nature 489: 57-74.

29. Bar-Joseph Z, Gitter A, Itamar S (2012) Studying and modeling dynamic biological processes using time-series gene expression data. Nature Reviews Genetics 13: 552-564.

30. Dusek JA, Otu HH, Wohlhueter AL, Bhasin M, Zerbini LF, et al. (2008) Genomic counter-stress changes induced by the relaxation response. PLoS One 3 : e2576.

31. Cozzolino M, Tagliaferri R, Castiglione S, Fortino V, Cicatelli A, et al. (2014 The creative psychosocial and cultural genomic healing experience: A new top-down epigenomic psychotherapeutic protocol. The International Journal of Psychosocial Genomics: Consciousness and Health Research. 1(1): 18-26.

32. Lichtenberg P, Bachner-Melman R, Gritsenko I, Ebstein R (2000) Exploratory Association Study between catechol-O-methyltransferase (COMT) high/low enzyme activity polymorphism and Hypnotizability. Am J. Med Genet 96: 771774

33. Lichtenberg P, Bachner-Melman R, Ebstein R, Crawford H (2004) Hypnotic Susceptibility: Multidimensional Relationships with Cloninger's Tridimensional Personality Questionnaire, COMT polymorphisms, absorption, and attentional characteristics. Int J Clin Exp Hypn 52: 47-72.

34. Creswell J, Irwin M, Burklund L, Lieberman M, Arevalo J et al. (2012) Mindfulness-Based Stress Reduction training reduces loneliness and proinflammatory gene expression in older adults: A small randomized controlled trial. Brain Behavlmmun 26: 1095-1101.

35. Sliwinski J, Elkins GR (2013) Enhancing placebo effects: insights from socia psychology.Am J ClinHypn 55: 236-248.

36. Cole S (2009) Social regulation of human gene expression. Current Directions in Psychological Science 18: 132-137.

37. Cole SW, Yan W, Galic Z, Arevalo J, Zack JA (2005) Expression-based monitoring of transcription factor activity: the TELiS database. Bioinformatics 21: 803-810.

38. Cole S, Hawkley L, Arevalo J, Sung C, Rose R, et al. (2007) Social regulation of gene expression in human leukocytes. Genome Biol 8: R189-R189.13.

39. Cole S, Arevalo J, Takahashi R, Sloan EK, Lutgendorf S, Sood A et al. (2010) Computational identification of gene-social environment interaction at the human IL6 locus. Proc Natl Acad Sci 107: 5681-5686.

40. Cole SW, Hawkley LC, Arevalo JM, Cacioppo JT (2011) Transcript origin analysis identifies antigen-presenting cells as primary targets of socially regulated gene expression in leukocytes. Proc Natl Acad Sci U S A 108: 30803085

41. Lavretsky H, Epel E Siddarth P, Nazarian N, Cyr N et al. (2013) A pilot study of yogic meditation for family dementia caregivers with depressive symptoms effects on mental health, cognition, and telomerase activity. Int. J Geriatric Psychiatry 28: 57-65.

42. Unternaehrer E, Luers $P$, Mill J, Dempster E, Meyer A, Staehli S, et al. (2012) Dynamic changes in DNA methylation of stress-associated genes (OXTR, BDNF) after acute psychosocial stress. Translational Psychiatry 2: e150.

43. Insel TR (2009) Disruptive insights in psychiatry: transforming a clinical discipline.J Clin Invest 119: 700-705.

44. Insel TR (2010) Faulty circuits. Sci Am 302: 44-51.

45. Insel TR (2012) Next-generation treatments for mental disorders.SciTransl Med 4: $155 \mathrm{ps} 19$

46. Rizzolatti G and Sinigaglia C (2008) Mirrors in the Brain: How our Minds Share Actions and Emotions. NY: Oxford University Press.

47. Grodzinsky Y, Nelken I (2014) Neuroscience. The neural code that makes us human Science 343: 978-979.

48. lacoboni M (2007) Face to face: The Neural Basis of Social Mirroring and Empathy: Psychiatric Annals. 37: 236-241.

49. lacoboni M (2008) Mirroring People: The new science of how we connect with others. NY: Farrar, Straus and Giroux.

50. Regev M, Honey CJ, Simony E, Hasson U (2013) Selective and invariant neural responses to spoken and written narratives. J Neurosci 33: 15978-15988.

51. Saey T (2010) First songbird genome arrives. Science News 177: 16

52. Warren W, Clayton D, Ellegren H, Arnold AP, Hillier LW et al. (2010) The genome of a songbird. Nature 464: 757-762.

53. Clayton DF (2013) Genomics of memory and learning in songbirds. Annu Rev Genomics Hum Genet 14: 45-65. 
Citation: Rossi EL, Rossi KL (2014) Quantum Perspectives of Consciousness, Cognition and Creativity:The Dirac Equation in a New Contour Integral Model of Brain Plasticity. J Appl Computat Math 3: 183. doi:10.4172/2168-9679.1000183

54. Drnevich J, Replogle KL, Lovell P, Hahn TP, Johnson F, et al. (2012) Impact of experience-dependent and -independent factors on gene expression in songbird brain. Proc Natl Acad Sci U S A 109 Suppl 2: 17245-17252.

55. Gunaratne PH1, Lin YC, Benham AL, Drnevich J, Coarfa C, et al. (2011) Song exposure regulates known and novel microRNAs in the zebra finch auditory forebrain. BMC Genomics 12: 277.

56. Morell V (2014) When the bat sings. Science 344: 1334-1337.

57. Bohn K Smarsh G, Smotherman M (2013) Social context evokes rapid changes in bat song syntax. Animal Behaviour 85: 1485-1491.

58. Knörnschild M (2014) Vocal production learning in bats.CurrOpinNeurobio 28C: $80-85$.

59. Susskind L, Friedman A (2014) Quantum Mechanics: The Theoretical Minimum NY: Basic Books.

60. Leslie M (2013) Cell Biology. NIH effort gambles on mysterious extracellular RNAs. Science 341: 947.

61. Pollard KS, Salama SR, Lambert N, Lambot MA, Coppens S, et al. (2006) An RNA gene expressed during cortical development evolved rapidly in humans. Nature 443: 167-172.

62. Hadamard J (1945) An essay on the psychology of invention in the mathematical field. NY: Princeton University Press.

63. Hameroff S and Penrose R (1996) Orchestrated reduction of quantum coherence in brain microtubules: A model for consciousness. Toward a Science of Consciousness-The First Tucson Discussions and Debates, Cambridge, MA: MIT Press.

64. Jeong H, Lim Y, Kim MS (2014) Coarsening measurement references and the quantum-to-classical transition. Phys Rev Lett 112: 010402.

65. Blinder S (2014) Fundamental Commutation Relations in Quantum Mechanics. Wolfram Demonstrations Project.

66. Rossi E, Rossi K (2011) Decoding the Chalmers Hard Problem of Consciousness: Qualia of the Molecular Biology of Creativity and Thought. Journal of Cosmology, Consciousness and the Universe: Quantum Physics, Evolution, Brain and Mind 210-227.

67. Mutze U (2014) Relativistic Quantum Dynamics in 1D and the Klein Paradox. Wolfram Demonstrations Project.
68. Gregory B (2010) The Expanding Role of Creativity in the Hypnotherapeutic Treatment of Resistance: An Integration of Erickson's Resistance Protocol with Principles of Physics and Classical Music Composition Theory. European Journal of Clinical Hypnosis 10: 42-57.

69. Hasson U, Honey CJ (2012) Future trends in Neuroimaging: Neural processes as expressed within real-life contexts. Neuroimage 62: 1272-1278.

70. Rembold J (2009) Quantum Notes, Ebook. New Mexico: New Mexico Tech.

71. Rossi E (2000) Dreams, Consciousness and Spirit: The Quantum Experience of Self-Reflection and Co-Creation. (3rd edn). NY: Zeig, Tucker, Theisen.

72. Xie L, Kang H, Xu Q, Chen MJ, Liao Y, et al. (2013) Sleep drives metabolite clearance from the adult brain. Science 342: 373-377.

73. Lu T, Aron L, Zullo J, Pan Y, Kim H, et al. (2014) REST and stress resistance in ageing and Alzheimer's disease. Nature 507: 448-454.

74. Tsai LH, Madabhushi R (2014) Alzheimer's disease: A protective factor for the ageing brain. Nature 507: 439-440.

75. Abbott A (2014) Row hits flagship brain plan. Human Brain Project, Nature 511 133-134.

76. Nature Editorial (2014) Brain fog: The criticism of Europe's Human Brain Project by leading scientists reflects a messy management structure that is in urgent need of clear direction. Nature.

77. Holmes EA, Craske MG, Graybiel AM (2014) Psychological treatments: A cal for mental-health science. Nature 511: 287-289.

78. Reardon S (2014) Gene-hunt gain for mental health. Nature 511: 393.

79. Vedral V (2012) Decoding Reality: The Universe as Quantum Information. NY Oxford University Press.

80. Rossi E (1997a) TheFeigenbaum Scenario in a Unified Science of Life and Mind. World Futures: 50: 633-645.

81. Rossi E (1997b) The symptom path to enlightenment: The psychobiology of Jung's Constructive Method. Psychological Perspectives 36: 68-84.

82. Rossi EL (1998) The Feigenbaum scenario as a model of the limits of conscious information processing. Biosystems 46: 113-122.

83. Rossi E (1999) The co-creative dynamics of dreams, consciousness and choice. Psychological Perspectives. 38: 116-127.

84. Doxiadis A, Papadimitriou C (2009) Logicomix: An Epic Search for Truth. NY: Bloomsbury. 